\title{
Computation of positive realizations for descriptor linear continuous- time systems
}

JEL: L91 DOI: 10.24136/atest.2018.428

Data zgłoszenia: 19.11.2018 Data akceptacji: 15.12.2018

A new method for computation of positive realizations of given transfer matrices of descriptor linear continuous-time linear systems is proposed. Necessary and sufficient conditions for the existence of positive realizations of transfer matrices are given. A procedure for computation of the positive realizations is proposed and illustrated by examples.

Keywords: computation, positive, realization, transfer matrix, descriptor, linear, continuous-time, system.

\section{Introduction}

A dynamical system is called positive if its trajectory starting from any nonnegative initial state remains forever in the positive orthant for all nonnegative inputs. An overview of state of the art in positive systems theory is given in the monographs [2, 13]. Variety of models having positive behavior can be found in engineering, economics, social sciences, biology and medicine, etc. [2, 13].

The determination of the matrices $A, B, C, D$ of the state equations of linear systems for given their transfer matrices is called the realization problem. The realization problem is a classical problem of analysis of linear systems and has been considered in many books and papers [4-6, 11, 12, 22, 24]. A tutorial on the positive realization problem has been given in the paper [1] and in the books $[2,13,24]$. The positive minimal realization problem for linear systems without and with delays has been analyzed in [3, 7-9, 13-17, $20,21,23]$. The existence and determination of the set of Metzler matrices for given stable polynomials have been considered in [10]. The realization problem for positive 2D hybrid systems has been addressed in [19]. For fractional linear systems the realization problem has been considered in $[4,18,22,24]$.

In this paper a new method for determination of positive realizations of descriptor linear continuous-time systems is proposed.

The paper is organized as follows. In section 2 some definitions and theorems concerning the positive continuous-time linear systems are recalled. A new method for determination of positive realizations for single-input single-output linear systems is proposed in section 3 and for multi-input multi-output systems in section 4. Concluding remarks are given in section 5 .

The following notation will be used: $\mathfrak{R}$ - the set of real numbers, $\mathfrak{R}^{n \times m}$ - the set of $n \times m$ real matrices, $\mathfrak{R}_{+}^{n \times m}$ - the set of $n \times m$ real matrices with nonnegative entries and $\mathfrak{R}_{+}^{n}=\mathfrak{R}_{+}^{n \times 1}$, $M_{n}$ - the set of $n \times n$ Metzler matrices (real matrices with nonnegative off-diagonal entries), $I_{n}$ - the $n \times n$ identity matrix.

\section{Preliminaries}

Consider the continuous-time linear system

$$
\begin{gathered}
\dot{x}(t)=A x(t)+B u(t), \\
y(t)=C x(t)+D u(t),
\end{gathered}
$$

where $x(t) \in \mathfrak{R}^{n}, \quad u(t) \in \mathfrak{R}^{m}, y(t) \in \mathfrak{R}^{p}$ are the state, input and output vectors and $A \in \mathfrak{R}^{n \times n}, \quad B \in \mathfrak{R}^{n \times m}$, $C \in \mathfrak{R}^{p \times n}, D \in \mathfrak{R}^{p \times m}$.

Definition 2.1. [2, 13] The system (2.1) is called (internally) positive if $x(t) \in \mathfrak{R}_{+}^{n}$ and $y(t) \in \mathfrak{R}_{+}^{p}, t \geq 0$ for any initial conditions $x(0) \in \mathfrak{R}_{+}^{n}$ and all inputs $u(t) \in \mathfrak{R}_{+}^{m}, t \geq 0$.

Theorem 2.1. [2, 13] The system (2.1) is positive if and only if

$$
A \in M_{n}, B \in \mathfrak{R}_{+}^{n \times m}, C \in \mathfrak{R}_{+}^{p \times n}, D \in \mathfrak{R}_{+}^{p \times m} .
$$

The transfer matrix of the system (2.1) is given by

$$
T(s)=C\left[I_{n} s-A\right]^{-1} B+D .
$$

The transfer matrix is called proper if

$$
\lim _{s \rightarrow \infty} T(s)=D \in \mathfrak{R}_{+}^{p \times m}
$$

and it is called strictly proper if $D=0$.

Definition 2.2. [1, 24] The matrices (2.2) are called a positive realization of $T(s)$ if they satisfy the equality (2.3).

Definition 2.3. [1, 24] The matrices (2.2) are called asymptotically stable if the matrix $A$ is an asymptotically stable Metzler matrix (Hurwitz Metzler matrix).

Theorem 2.2. [1, 24] The positive realization (2.2) is asymptotically stable if and only if all coefficients of the polynomial

$$
p_{A}(s)=\operatorname{det}\left[I_{n} s-A\right]=s^{n}+a_{n-1} s^{n-1}+\ldots+a_{1} s+a_{0}
$$

are positive, i.e. $a_{i}>0$ for $i=0,1, \ldots, n-1$.

The positive realization problem can be stated as follows. Given a proper transfer matrix $T(s)$ find its positive realization (2.2).

Theorem 2.3. [24] If (2.2) is a positive realization of (2.3) then the matrices

$$
\bar{A}=P A P^{-1}, \bar{B}=P B, \bar{C}=C P^{-1}, \bar{D}=D
$$
are also a positive realization of (2.3) if and only if the matrix $P \in \mathfrak{R}_{+}^{n \times n}$ is a monomial matrix (in each row and in each column only one entry is positive and the remaining entries are zero).

Proof. Proof follows immediately from the fact that $P^{-1} \in \mathfrak{R}_{+}^{n \times n}$ if and only if $P$ is a monomial matrix. $\square$

\section{Computation of positive realizations of descriptor single- input single-output systems}

Consider the descriptor continuous-time linear system

$$
\begin{gathered}
E \dot{x}(t)=A x(t)+B u(t) \\
y(t)=C x(t)
\end{gathered}
$$

where $x(t) \in \mathfrak{R}^{n}, u(t) \in \mathfrak{R}^{m}, y(t) \in \mathfrak{R}^{p}$ are the state, input and output vectors and $E, A \in \mathfrak{R}^{n \times n}, B \in \mathfrak{R}^{n \times m}$, $C \in \mathfrak{R}^{p \times n}, D \in \mathfrak{R}^{p \times m}$.

It is assumed that $\operatorname{det} E=0$ and the pencil of $(E, A)$ is regular, i.e. 
$\operatorname{det}[E s-A] \neq 0$ for some $s \in \mathbf{C}$ (the field of complex numbers).

Definition 3.1. The descriptor system (3.1) is called (internally) positive if $x(t) \in \mathfrak{R}_{+}^{n}, y(t) \in \mathfrak{R}_{+}^{p}, t \geq 0$ for any consistent initial conditions $\quad x(0) \in \mathfrak{R}_{+}^{n} \quad$ and all inputs $u^{(k)}(t)=\frac{d^{k} u(t)}{d t^{k}} \in \mathfrak{R}_{+}^{m}$ for $t \geq 0$ and $k=0,1, \ldots, q$.

The transfer matrix of the system (3.1)

$$
T(s)=C[E s-A]^{-1} B \in \mathfrak{R}^{p \times m}(s)
$$

can be decomposed in the polynomial part $P(s)$ and strictly proper part $T_{s p}(s)$, i.e.

$$
T(s)=P(s)+T_{s p}(s),
$$

where

$$
P(s)=P_{0}+P_{1} s+\ldots+P_{q} s^{q} \in \mathfrak{R}^{p \times m}[s]
$$

and

$$
T_{s p}(s)=\bar{C}\left[I_{n} s-\bar{A}\right]^{-1} \bar{B} .
$$

First the new method for computation of a positive realization of given transfer function will be presented.

Theorem 3.1. There exists the positive realization

$$
\bar{A}=\left[\begin{array}{cccccc}
s_{1} & 0 & 0 & \cdots & 0 & 0 \\
1 & s_{2} & 0 & \cdots & 0 & 0 \\
\vdots & \vdots & \vdots & \ddots & \vdots & \vdots \\
0 & 0 & 0 & \cdots & s_{n-1} & 0 \\
0 & 0 & 0 & \cdots & 1 & s_{n}
\end{array}\right], \bar{B}=\left[\begin{array}{c}
b_{1} \\
b_{2} \\
\vdots \\
b_{n}
\end{array}\right], \bar{C}=\left[\begin{array}{llll}
0 & \cdots & 0 & 1
\end{array}\right]
$$

of the transfer function

$$
T_{s p}(s)=\frac{\bar{m}_{n-1} s^{n-1}+\ldots+\bar{m}_{1} s+\bar{m}_{0}}{s^{n}+d_{n-1} s^{n-1}+\ldots+d_{1} s+d_{0}}
$$

if and only if

$$
\bar{B}=\left[\begin{array}{c}
b_{1} \\
b_{2} \\
\vdots \\
b_{n}
\end{array}\right]=\left[\begin{array}{ccccc}
1 & s_{1} & s_{1} s_{2} & \cdots & s_{1} s_{2} \ldots s_{n-1} \\
0 & 1 & s_{1}+s_{2} & \cdots & s_{1}+s_{2}+\ldots+s_{n-1} \\
\vdots & \vdots & \vdots & \ddots & \vdots \\
0 & 0 & 0 & \cdots & 1
\end{array}\right]^{-1}\left[\begin{array}{c}
\bar{m}_{0} \\
\bar{m}_{1} \\
\vdots \\
\bar{m}_{n-1}
\end{array}\right] \in \mathfrak{R}_{+}^{n},
$$

where $s_{k}, k=1, \ldots, n$ are the zeros of the denominator

$$
d(s)=s^{n}+d_{n-1} s^{n-1}+\ldots+d_{1} s+d_{0}=\left(s+s_{1}\right)\left(s+s_{2}\right) \ldots\left(s+s_{n}\right) .
$$

Proof. The proof is given in [6].

Remark 3.1. The positive realization (3.6) is asymptotically stable if and only if all coefficients of the denominator (3.9) are positive, i.e. $d_{k}>0, k=0,1, \ldots, n-1[6]$.

Theorem 3.1 and Remark 3.1 can be easily extended to the multiinput multi-output linear systems [6].

Example 3.1. Compute the positive realization (3.6) of the transfer function

$$
T_{s p}(s)=\frac{\bar{m}_{2} s^{2}+\bar{m}_{1} s+\bar{m}_{0}}{s^{3}+d_{2} s^{2}+d_{1} s+d_{0}}=\frac{s^{2}+4 s+7}{s^{3}+6 s^{2}+11 s+6} .
$$

The denominator $d(s)=s^{3}+6 s^{2}+11 s+6=(s+1)(s+2)(s+3)$ has the real zeros $\bar{s}_{1}=-1, \bar{s}_{2}=-2, \bar{s}_{3}=-3$ and the matrix $A$ is Hurwitz of the form

$$
\bar{A}=\left[\begin{array}{ccc}
s_{1} & 0 & 0 \\
1 & s_{2} & 0 \\
0 & 1 & s_{3}
\end{array}\right]=\left[\begin{array}{ccc}
-1 & 0 & 0 \\
1 & -2 & 0 \\
0 & 1 & -3
\end{array}\right] .
$$

Using (3.8) and (3.10) we obtain

$$
\bar{B}=\left[\begin{array}{ccc}
1 & s_{1} & s_{1} s_{2} \\
0 & 1 & s_{1}+s_{2} \\
0 & 0 & 1
\end{array}\right]^{-1}\left[\begin{array}{c}
\bar{m}_{0} \\
\bar{m}_{1} \\
\bar{m}_{2}
\end{array}\right]=\left[\begin{array}{lll}
1 & 1 & 2 \\
0 & 1 & 3 \\
0 & 0 & 1
\end{array}\right]^{-1}\left[\begin{array}{l}
7 \\
4 \\
1
\end{array}\right]=\left[\begin{array}{l}
4 \\
1 \\
1
\end{array}\right]
$$

and the matrix $\bar{C}$ has the form

$$
\bar{C}=\left[\begin{array}{lll}
0 & 0 & 1
\end{array}\right] .
$$

The positive asymptotically stable realization of $(3.10)$ is given by (3.11) - (3.13).

It is easy to check that the matrices

$$
\hat{A}=\left[\begin{array}{ccc}
-1 & 1 & 0 \\
0 & -2 & 1 \\
0 & 0 & -3
\end{array}\right], \hat{B}=\left[\begin{array}{l}
0 \\
0 \\
1
\end{array}\right], \hat{C}=\left[\begin{array}{lll}
4 & 1 & 1
\end{array}\right]
$$

are also the positive asymptotically stable realization of the transfer function (3.10).

Theorem 3.2. If the matrices (3.6) are a positive realization of the strictly proper transfer function (3.7) then the matrices

$$
\begin{aligned}
E & =\left[\begin{array}{cccccc}
I_{n} & 0 & 0 & \cdots & 0 & 0 \\
0 & 0 & 0 & \cdots & 0 & 0 \\
0 & 1 & 0 & \cdots & 0 & 0 \\
\vdots & \vdots & \vdots & \ddots & \vdots & \vdots \\
0 & 0 & 0 & \cdots & 1 & 0
\end{array}\right] \in \Re_{+}^{\bar{n} \times \bar{n}}, A=\left[\begin{array}{cccccc}
\bar{A} & \bar{B} & 0 & \cdots & 0 & 0 \\
0 & -1 & 0 & \cdots & 0 & 0 \\
0 & 0 & 1 & \cdots & 0 & 0 \\
\vdots & \vdots & \vdots & \ddots & \vdots & \vdots \\
0 & 0 & 0 & \cdots & 0 & 1
\end{array}\right] \in M_{\bar{n}} \\
B & =\left[\begin{array}{c}
0 \\
1 \\
0 \\
\vdots \\
0
\end{array}\right] \Re_{+}^{\bar{n} \times 1}, C=\left[\begin{array}{lllll}
\bar{C} & P_{0} & P_{1} & \cdots & P_{q}
\end{array}\right] \in \mathfrak{R}_{+}^{\mid \times \bar{n}}, \bar{n}=n+q+1
\end{aligned}
$$

are a positive realization of the transfer function (3.3) if and only if

$$
P_{k} \in \mathfrak{R}_{+} \text {for } k=0,1, \ldots, q \text {. }
$$

Proof. Using (3.15) it is easy to verify that

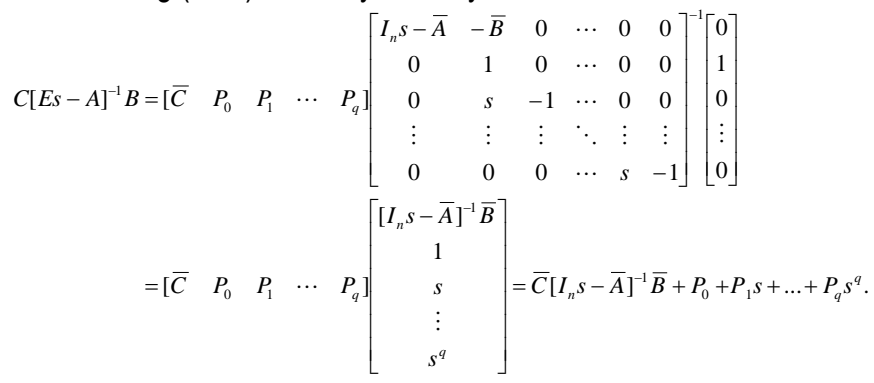

Therefore, the matrices (3.15) are the positive realization of the transfer function (3.3). $\square$

Remark 3.2. Note that $A \in M_{n}$ if $\bar{B} \in \mathfrak{R}_{+}^{n}$ and the condition (3.8) is satisfied.

Remark 3.3. The positive realization (3.15) is asymptotically stable if and only if the matrix $\bar{A} \in M_{n}$ is Hurwitz.

Example 3.2. Compute the positive realization (3.15) of the transfer function 


$$
T_{s p}(s)=\frac{3 s^{4}+20 s^{3}+46 s^{2}+44 s+19}{s^{3}+6 s^{2}+11 s+6} .
$$

The transfer function (3.18) can be decomposed as follows

$$
T(s)=P(s)+T_{s p}(s),
$$

where

$$
\begin{gathered}
P(s)=P_{0}+P_{1} s=2+3 s, \\
T_{s p}(s)=\frac{s^{2}+4 s+7}{s^{3}+6 s^{2}+11 s+6} .
\end{gathered}
$$

The positive realization of (3.19c) has been computed in Example 3.1 and has the form given by (3.11) - (3.13).

The conditions of Theorem 3.2 for the existence of positive realization are satisfied since the coefficients of (3.19b) are positive, i.e. $P_{0}=2, P_{1}=3$.

Therefore, by Theorem 3.2 the desired positive realization of the transfer function (3.18) has the form

$E=\left[\begin{array}{lllll}1 & 0 & 0 & 0 & 0 \\ 0 & 1 & 0 & 0 & 0 \\ 0 & 0 & 1 & 0 & 0 \\ 0 & 0 & 0 & 0 & 0 \\ 0 & 0 & 0 & 1 & 0\end{array}\right], A=\left[\begin{array}{ccccc}-1 & 0 & 0 & 4 & 0 \\ 1 & -2 & 0 & 1 & 0 \\ 0 & 1 & -3 & 1 & 0 \\ 0 & 0 & 0 & -1 & 0 \\ 0 & 0 & 0 & 0 & 1\end{array}\right]$

$$
B=\left[\begin{array}{l}
0 \\
0 \\
0 \\
1 \\
0
\end{array}\right], C=\left[\begin{array}{lllll}
0 & 0 & 1 & 2 & 3
\end{array}\right] .
$$

\section{Computation of positive realizations of descriptor MIMO} systems

In this section the method presented in section 3 will be extended to multi-input multi-output linear continuous-time (MIMO) systems.

The strictly proper transfer matrix (3.5) can be written in the form with common least row denominator

$$
\begin{gathered}
T_{s p}(s)=\left[\begin{array}{ccc}
\frac{\bar{m}_{11}(s)}{\bar{d}_{1}(s)} & \cdots & \frac{\bar{m}_{1 m}(s)}{\bar{d}_{1}(s)} \\
\vdots & \ddots & \vdots \\
\bar{m}_{p 1}(s) & \cdots & \bar{m}_{p m}(s) \\
\frac{\bar{d}_{p}(s)}{\bar{d}_{p}(s)} & \cdots & \bar{m}_{i k}(s)=\bar{m}_{i k n-1} s^{n-1}+\ldots+\bar{m}_{i k 1} s+\bar{m}_{i k 0}, \\
\bar{d}_{i}(s)=s^{n}+\bar{d}_{i n-1} s^{n-1}+\ldots+\bar{d}_{i 1} s+\bar{d}_{i 0}, i=1, \ldots, p ; k=1, \ldots, m
\end{array}\right.
\end{gathered}
$$

or with common least column denominator

$$
\begin{gathered}
T_{s p}(s)=\left[\begin{array}{ccc}
\frac{\hat{m}_{11}(s)}{\bar{d}_{1}(s)} & \cdots & \frac{\hat{m}_{1 m}(s)}{\bar{d}_{m}(s)} \\
\vdots & \ddots & \vdots \\
\hat{m}_{p 1}(s) & \cdots & \frac{\hat{m}_{p m}(s)}{\hat{d}_{1}(s)} \\
\hat{d}_{m}(s)
\end{array}\right], \hat{m}_{i k}(s)=\hat{m}_{i k n-1} s^{n-1}+\ldots+\hat{m}_{i k 1} s+\hat{m}_{i k 0}, \\
\hat{d}_{k}(s)=s^{n}+\hat{d}_{k n-1} s^{n-1}+\ldots+\hat{d}_{k 1} s+\hat{d}_{k 0}, i=1, \ldots, p ; k=1, \ldots, m .
\end{gathered}
$$

Further we shall consider in details only the first case (4.1) since the considerations for (4.2) are similar (dual).
The matrix $\bar{A}$ of the desired realization has the form

$$
\bar{A}=\operatorname{blockdiag}\left[\bar{A}_{1} \quad \cdots \quad \bar{A}_{p}\right],
$$

where

$$
\bar{A}_{i}=\left[\begin{array}{cccccc}
s_{i 1} & 0 & 0 & \cdots & 0 & 0 \\
1 & s_{i 2} & 0 & \cdots & 0 & 0 \\
\vdots & \vdots & \vdots & \ddots & \vdots & \vdots \\
0 & 0 & 0 & \cdots & s_{i n-1} & 0 \\
0 & 0 & 0 & \cdots & 1 & s_{i n}
\end{array}\right] \in M_{n_{i}}, i=1, \ldots, p .
$$

The matrix $\bar{B}$ has the form

$$
\bar{B}=\left[\begin{array}{ccc}
\bar{B}_{11} & \cdots & \bar{B}_{1 m} \\
\vdots & \ddots & \vdots \\
\bar{B}_{p 1} & \cdots & \bar{B}_{p m}
\end{array}\right] \in \mathfrak{R}_{+}^{n p \times m}, \bar{B}_{i k}=\left[\begin{array}{c}
b_{i k 1} \\
b_{i k 2} \\
\vdots \\
b_{i k k_{i}}
\end{array}\right], i=1, \ldots, p, k=1, \ldots, m
$$

The entries of the matrices $\bar{B}_{i k}$ are computed in the same way as of the matrix $\bar{B}$ in section 3 using the equation

$$
B_{i}=S_{i}^{-1} M_{i} \in \mathfrak{R}_{+}^{n_{i}}, i=1, \ldots, p,
$$

where

$$
\begin{gathered}
S_{i}=\left[\begin{array}{ccccc}
1 & s_{i 1} & s_{i 1} s_{i 2} & \ldots & s_{i 1} s_{i 2} \ldots s_{i n-1} \\
0 & 1 & s_{i 1}+s_{i 2} & \cdots & s_{i 1}+s_{i 2}+\ldots+s_{i n-1} \\
\vdots & \vdots & \vdots & \ddots & \vdots \\
0 & 0 & 0 & \cdots & 1
\end{array}\right], i=1, \ldots, p, \\
M_{i}=\left[\begin{array}{c}
\bar{m}_{i k 0} \\
\bar{m}_{i k 1} \\
\vdots \\
\bar{m}_{i k n_{i-1}}
\end{array}\right], i=1, \ldots, p, k=1, \ldots, m . \quad \text { (4.5c) }
\end{gathered}
$$

The matrix $\bar{C}$ is given by

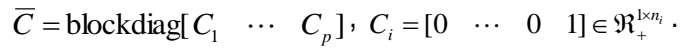

Theorem 4.1. If the matrices (4.3), (4.4) and (4.6) are a positive realization of the strictly proper transfer matrix (4.1) then the matrices

$$
\begin{gathered}
\tilde{E}=\left[\begin{array}{cccccc}
I_{n} & 0 & 0 & \cdots & 0 & 0 \\
0 & 0 & 0 & \cdots & 0 & 0 \\
0 & I_{m} & 0 & \cdots & 0 & 0 \\
\vdots & \vdots & \vdots & \ddots & \vdots & \vdots \\
0 & 0 & 0 & \cdots & I_{m} & 0
\end{array}\right] \in \Re_{+}^{\bar{n} \times \bar{n}}, \tilde{A}=\left[\begin{array}{cccccc}
\bar{A} & \bar{B} & 0 & \cdots & 0 & 0 \\
0 & -I_{m} & 0 & \cdots & 0 & 0 \\
0 & 0 & I_{m} & \cdots & 0 & 0 \\
\vdots & \vdots & \vdots & \ddots & \vdots & \vdots \\
0 & 0 & 0 & \cdots & 0 & I_{m}
\end{array}\right] \in M_{\bar{n}} \\
\tilde{B}=\left[\begin{array}{c}
0 \\
I_{m} \\
0 \\
\vdots \\
0
\end{array}\right] \in \Re_{+}^{\bar{n} \times m}, C=\left[\begin{array}{lllll}
\bar{C} & P_{0} & P_{1} & \cdots & P_{q}
\end{array}\right] \in \Re_{+}^{p \times \bar{n}}, \bar{n}=n+(q+1) m
\end{gathered}
$$

are a positive realization of the transfer matrix (3.3) if and only if

$$
P_{k} \in \mathfrak{R}_{+}^{p \times m} \text { for } k=0,1, \ldots, q .
$$

Proof. The proof is similar to the proof of Theorem 3.2. 
From the above considerations we have the following procedure for computation of the positive realization (4.7) of the given transfer matrix $T(s)$.

\section{Procedure 4.1.}

Step 1. Decompose the given matrix $T(s)$ in the polynomial part (3.4b) and strictly proper part (3.5).

Step 2. Compute the zeros $s_{i j}, i=1, \ldots, p, j=1, \ldots, n_{j}$ of the denominator $d_{i}(s), i=1, \ldots, p$ and find the matrices (4.3b), (4.3a).

Step 3. Using (4.5b) and (4.5c) compute the matrices $S_{i}, M_{i}$ and check the conditions (4.5a). If the conditions (4.5a) are satisfied then there exists $\bar{B} \in \mathfrak{R}_{+}^{n p \times m}$ and the positive realization of $T(s)$.

The desired positive realization is given by (4.7).

Example 4.1. Compute the positive realization (4.7) of the transfer matrix

$$
T(s)=\left[\begin{array}{c}
\frac{2 s^{3}+7 s^{2}+7 s+2}{s^{2}+3 s+2} \\
\frac{3 s^{2}+11 s+6}{s+3}
\end{array}\right] .
$$

Using Procedure 4.1 we obtain the following.

Step 1. The matrix (4.9) can be decomposed in the polynomial part

$$
P(s)=\left[\begin{array}{l}
2 \\
3
\end{array}\right] s+\left[\begin{array}{l}
1 \\
2
\end{array}\right]
$$

and strictly proper part

$$
T_{s p}(s)=\left[\begin{array}{c}
\frac{2 s+3}{s^{2}+3 s+2} \\
\frac{1}{s+3}
\end{array}\right] .
$$

Step 2. The zeros of the first denominator

$$
d_{1}(s)=s^{2}+3 s+2
$$

are: $s_{11}-1, s_{12}-2$ and of the second denominator

$$
d_{2}(s)=s+3
$$

$s_{21}=-3$.

Therefore, the matrix $\bar{A}$ has the form

$$
\bar{A}=\left[\begin{array}{cc}
\bar{A}_{1} & 0 \\
0 & \bar{A}_{2}
\end{array}\right]=\left[\begin{array}{ccc}
-1 & 0 & 0 \\
1 & -2 & 0 \\
0 & 0 & -3
\end{array}\right] .
$$

Step 3. In this case

$$
\bar{B}=\left[\begin{array}{l}
\bar{B}_{1} \\
\bar{B}_{2}
\end{array}\right], \bar{B}_{1}=\left[\begin{array}{l}
b_{11} \\
b_{12}
\end{array}\right], \bar{B}_{2}=b_{21}
$$

and using (4.5a) we obtain

$$
\bar{B}_{1}=\left[\begin{array}{cc}
1 & s_{11} \\
0 & 1
\end{array}\right]^{-1}\left[\begin{array}{l}
\bar{m}_{10} \\
\bar{m}_{11}
\end{array}\right]=\left[\begin{array}{ll}
1 & 1 \\
0 & 1
\end{array}\right]\left[\begin{array}{l}
3 \\
2
\end{array}\right]=\left[\begin{array}{l}
5 \\
2
\end{array}\right]
$$

$$
\bar{B}_{2}=b_{21}=1 \text {. }
$$

Therefore, the matrix

$$
\bar{B}=\left[\begin{array}{l}
\bar{B}_{1} \\
\bar{B}_{2}
\end{array}\right]=\left[\begin{array}{l}
5 \\
2 \\
1
\end{array}\right]
$$

and the matrix

$$
\bar{C}=\left[\begin{array}{cc}
\bar{C}_{1} & 0 \\
0 & \bar{C}_{2}
\end{array}\right]=\left[\begin{array}{lll}
0 & 1 & 0 \\
0 & 0 & 1
\end{array}\right] .
$$

The desired positive realization of (4.9) is given by

$$
\begin{aligned}
& \widetilde{E}=\left[\begin{array}{lllll}
1 & 0 & 0 & 0 & 0 \\
0 & 1 & 0 & 0 & 0 \\
0 & 0 & 1 & 0 & 0 \\
0 & 0 & 0 & 0 & 0 \\
0 & 0 & 0 & 1 & 0
\end{array}\right], \tilde{A}=\left[\begin{array}{ccccc}
-1 & 0 & 0 & 5 & 0 \\
1 & -2 & 0 & 2 & 0 \\
0 & 0 & -3 & 1 & 0 \\
0 & 0 & 0 & -1 & 0 \\
0 & 0 & 0 & 0 & 1
\end{array}\right] \\
& \widetilde{B}=\left[\begin{array}{l}
0 \\
0 \\
0 \\
1 \\
0
\end{array}\right], \tilde{C}=\left[\begin{array}{lllll}
0 & 1 & 0 & 2 & 1 \\
0 & 0 & 1 & 3 & 2
\end{array}\right] \text {. }
\end{aligned}
$$

Now let us consider the strictly proper transfer matrix (4.11) as the matrix with least common column denominator

$$
T_{s p}(s)=\frac{1}{d(s)}\left[\begin{array}{c}
2 s^{2}+9 s+9 \\
s^{2}+3 s+2
\end{array}\right]
$$

where

$$
d(s)=\left(s^{2}+3 s+3\right)(s+3)=s^{3}+6 s^{2}+12 s+9
$$
has the zeros: $s_{1}=-1, s_{2}=-2, s_{3}=-3$.

Therefore, the matrix $\bar{A}$ has the form

$$
\bar{A}=\left[\begin{array}{ccc}
-1 & 1 & 0 \\
0 & -2 & 1 \\
0 & 0 & -3
\end{array}\right]
$$

In this case the matrix $\bar{B}$ is given by

$$
\bar{B}=\left[\begin{array}{l}
0 \\
0 \\
1
\end{array}\right] \text {. }
$$

Using the dual method to the method for computation of the matrix $\bar{B}$ we obtain

$$
\bar{C}=\left[\begin{array}{lll}
2 & 3 & 2 \\
0 & 0 & 1
\end{array}\right]
$$

Therefore, the desired positive realization of (4.9) has the form 


$$
\begin{gathered}
\hat{E}=\left[\begin{array}{ccccc}
1 & 0 & 0 & 0 & 0 \\
0 & 1 & 0 & 0 & 0 \\
0 & 0 & 1 & 0 & 0 \\
0 & 0 & 0 & 0 & 0 \\
0 & 0 & 0 & 1 & 0
\end{array}\right], \hat{A}=\left[\begin{array}{ccccc}
-1 & 1 & 0 & 0 & 0 \\
0 & -2 & 1 & 0 & 0 \\
0 & 0 & -3 & 1 & 0 \\
0 & 0 & 0 & -1 & 0 \\
0 & 0 & 0 & 0 & 1
\end{array}\right] \\
\hat{B}=\left[\begin{array}{l}
0 \\
0 \\
0 \\
1 \\
0
\end{array}\right], \hat{C}=\left[\begin{array}{lllll}
2 & 3 & 2 & 2 & 1 \\
0 & 0 & 1 & 3 & 2
\end{array}\right] .
\end{gathered}
$$

\section{Concluding remarks}

A new method for determination of positive realizations of transfer matrices of descriptor linear continuous-time systems has been proposed. Necessary and sufficient conditions for the existence of the positive realizations have been established (Theorems 3.1, 3.2 and 4.1). A procedure for computation of the positive realizations has been proposed and illustrated by an example (Example 4.1). The presented method can be extended to descriptor linear discrete-time systems and to descriptor linear fractional systems.

\section{References}

1. Benvenuti L., Farina L.: A tutorial on the positive realization problem. IEEE Trans. on Automatic Control, vol. 49, no. 5, 2004, 651-664.

2. Farina L., Rinaldi S.: Positive Linear Systems; Theory and Applications. J. Wiley, New York, 2000.

3. Kaczorek T.: A modified state variable diagram method for determination of positive realizations of linear continuous-time systems with delays. Int. J. Appl. Math. Comput. Sci., vol. 22, no. 4, 2012, 897-905.

4. Kaczorek T.: A new method for computation of positive realizations of fractional linear continuous-time systems, 2017.

5. Kaczorek T.: A new method for computation of positive realizations of linear discrete-time systems, 2017.

6. Kaczorek T.: A new method for determination of positive realizations of linear continuous-time systems, 2017.

7. Kaczorek T.: A realization problem for positive continuous-time linear systems with reduced numbers of delays. Int. J. Appl. Math. Comput. Sci., vol. 16, no. 3, 2006, 325-331.

8. Kaczorek T.: Computation of positive stable realizations for linear continuous-time systems. Bull. Pol. Acad. Techn. Sci., vol. 59, no. 3, 2011, 273-281.

9. Kaczorek T.: Computation of realizations of discrete-time cone systems. Bull. Pol. Acad. Sci. Techn., vol. 54, no. 3, 2006, 347350.

10. Kaczorek T.: Existence and determination of the set of Metzler matrices for given stable polynomials. Int. J. Appl. Math. Comput. Sci., vol. 22, no. 2, 2012, 389-399.
11. Kaczorek T.: Linear Control Systems: Analysis of Multivariable Systems. J. Wiley \& Sons, New York, 1992.

12. Kaczorek T.: Polynomial and Rational Matrices. SpringerVerlag, London, 2007.

13. Kaczorek T.: Positive 1D and 2D Systems. Springer-Verlag, London, 2002.

14. Kaczorek T.: Positive minimal realizations for singular discretetime systems with delays in state and delays in control. Bull. Pol. Acad. Sci. Techn., vol. 53, no. 3, 2005, 293-298.

15. Kaczorek T.: Positive stable realizations of continuous-time linear systems. Proc. Conf. Int. Inf. and Eng. Syst., KrynicaZdrój, Poland, 17-21 September, 2012.

16. Kaczorek T.: Positive stable realizations for fractional descriptor continuous-time linear systems. Archives of Control Sciences, vol. 22, no. 3, 2012, 255-265.

17. Kaczorek T.: Positive stable realizations with system Metzler matrices. Archives of Control Sciences, vol. 21, no. 2, 2011, 167-188.

18. Kaczorek T.: Realization problem for fractional continuous-time systems. Archives of Control Sciences, vol. 18, no. 1, 2008, 4358.

19. Kaczorek T.: Realization problem for positive $2 \mathrm{D}$ hybrid systems. COMPEL, vol. 27, no. 3, 2008, 613-623.

20. Kaczorek T.: Realization problem for positive discrete-time systems with delays. System Science, vol. 30, no. 4, 2004, 117130.

21. Kaczorek T.: Realization problem for positive multivariable discrete-time linear systems with delays in the state vector and inputs. Int. J. Appl. Math. Comput. Sci., vol. 16, no. 2, 2006, 169-174.

22. Kaczorek T.: Selected Problems of Fractional Systems Theory. Springer-Verlag, 2011.

23. Kaczorek T., Busłowicz M.: Minimal realization for positive multivariable linear systems with delay. Int. J. Appl. Math. Comput. Sci., vol. 14, no. 2, 2004, 181-187.

24. Kaczorek T., Sajewski Ł.: Realization Problem for Positive and Fractional Systems, Springer, 2014

\section{Wyznaczanie dodatnich realizacji deskryptowych ciagłych układow liniowych}

W pracy podano nową metodę wyznaczania dodatnich realizacji dla zadanych macierzy transmitacji operatorowych deskryptowych( singularnych) ciagłych układów liniowych. Sformułowano warunki konieczne i wystarczajace istnienia dodatnich realizacji dla tej klasy deskryptowych ciagłych układów liniowych. Podano procedurę wyznaczania tych realizacji. Procedura ta została zlustrowana przykładami liczbowymi.

Autor

prof. dr hab. inż. Tadeusz Kaczorek - Białystok University of Technology, Faculty of Electrical Engineering, Wiejska 45D, 15-351 Białystok, e-mail: kaczorek@ee.pw.edu.pl 